\title{
Clinical and Pathologic Presentation of Primary Ocular Surface Tumors among Zambians
}

\author{
Peter Julius $^{a}$ Stepfanie N. Siyumbwa ${ }^{a}$ Phyllis Moongab $^{\text {Fred Maate }}{ }^{a}$ \\ Trevor Kaile $^{a}$ Guobin Kang ${ }^{c}$ John T. West ${ }^{d}$ Charles Wood ${ }^{c}$ \\ Peter C. Angeletti ${ }^{\mathrm{C}}$
}

aDepartment of Pathology and Microbiology, School of Medicine, Lusaka, Zambia; b University Teaching Hospital, Eye Hospital, Lusaka, Zambia; 'Nebraska Center for Virology and the School of Biological Sciences, University of Nebraska-Lincoln, Lincoln, NE, USA; d Nebraska Center for Virology and the Department of Biochemistry, University of Nebraska-Lincoln, Lincoln, NE, USA

\section{Keywords}

Ocular surface tumors - Ocular surface squamous neoplasia. Squamous cell carcinoma - Conjunctiva . Cornea

\begin{abstract}
Aim: This study aimed to characterize the clinical and pathologic presentation of ocular surface tumors (OSTs) and to more precisely differentiate the grades of ocular surface squamous neoplasia (OSSN) and benign lesions among Zambians. Methods: Two-hundred sixty-five Zambian patients presenting with ocular surface growths, suspicious for OSSN, were recruited between November 2017 and November 2019 to a cross-sectional study to investigate their lesions. Sociodemographic data were collected, HIV infection status and vision tests were performed, and lesions were measured and documented. Lesions $>2 \mathrm{~mm}$ in diameter were excised and sent for pathology analysis. In addition to the biopsies, tears, blood, and buccal swabs were collected. CD4+ T-cell counts were measured by flow cytometry. Lesions were classified according to the WHO guidelines. $x^{2}$ and bivariate correlations were used to analyze variable associations and strengths with phi/Cramer's V and correlation
\end{abstract}

(C) 2021 The Author(s)

Published by S. Karger AG, Basel

This is an Open Access article licensed under the Creative Commons Attribution-NonCommercial-4.0 International License (CC BY-NC) (http://www.karger.com/Services/OpenAccessLicense), applicable to the online version of the article only. Usage and distribution for commercial purposes requires written permission. coefficients, respectively. Binary logistics was used to adjust for covariance. Results: In this study, $68.3 \%$ of the participants were found to be HIV positive. The most frequent diagnoses were invasive OSSN (45.3\%), preinvasive OSSN (29.1\%), and pterygium (22.6\%). Invasive OSSN comprised keratinizing squamous cell carcinoma (SCC) $(87.5 \%)$, basaloid SCC (3.3\%), and spindle cell carcinoma (3.3\%). Unusual carcinomas, not described previously, included hybrid SCC (5.0\%) and acantholytic SCC (0.8\%). Invasive OSSN had advanced tumor (T3/T4) staging (93.3\%) at diagnosis. Lymphadenopathy was rare $(2.3 \%)$, and metastasis was absent. Patients were mostly female (59.2\%). Median age was 36 (interquartile ranges 33-41) years (ranges 18-81). Patients with invasive OSSN were more likely to present with pain $(p=$ $0.007)$, redness $(p=0.034)$, excessive tearing $(p=0.0001)$, discharge $(p=0.011)$, bleeding $(p=0.007)$, reduced vision $(p=0.0001)$, fungating lesion $(p=0.001)$, and blindness $(p=$ $0.005)$; location at temporal limbus $(p=0.0001)$, inferior limbus ( $p=0.0001)$, or circumlimbal $(p=0.001)$; and extension to cornea $(p=0.006)$ and forniceal palpebral conjunctiva $(p=0.001)$. Invasive OSSN was associated with any smoking habit and alcohol consumption ( $p=0.04$ and 0.03 , respectively). HIV positivity was strongly associated with OSSN 
(74.6\% OSSN vs. $49.3 \%$ benign lesions; $p=0.0001$; phi: 0.237 [ $p=0.0001])$. Conclusion: OSTs are very common in Zambia and are strongly associated with HIV coinfection. Patients with OSSN were more likely to be HIV positive than those with pterygia. Despite the commonality of OSTs in sub-Saharan Africa, these cancers have historically been poorly characterized.

(c) 2021 The Author(s)

Published by S. Karger AG, Basel

\section{Introduction}

Primary ocular surface tumors (OSTs) are diverse and include degenerative, inflammatory lesions, choristomas/hamartomas, benign and malignant epithelial lesions, melanocytic, hematolymphoid, and soft tissue tumors $[1,2]$. Most of these lesions have overlapping clinical features that make a distinction on clinical examination alone a challenge [3]. However, the behavior, prognosis, and treatment of these lesions vary, necessitating a precise histopathologic tissue diagnosis to determine the most appropriate management [4]. Type and frequency of these tumors vary depending on the population studied [3, 5-10]. Sub-Saharan Africa has seen a rising incidence of OSTs, mostly ocular surface squamous neoplasia (OSSN) associated with the HIV pandemic [3, 11, 12]. In low- and middle-income countries, most centers diagnose and treat OSTs based on clinical impression alone due to inadequate diagnostic pathology services $[3,13$, 14]. Because of this, there is a need to identify the clinicopathologic factors that can help distinguish individual disease states for appropriate management of patients in a setting with a high HIV burden.

This study was focused on determining the relative frequency of OSTs, their precursor lesions and variants, and to investigate the comparative clinicopathologic features between preinvasive and invasive OSSN versus benign lesions surgically removed from adult patients at the Eye Hospital associated with the University Teaching Hospitals (UTHs), in Zambia.

\section{Methods}

A cross-sectional study was used to investigate OST at the UTH, Eye Hospital in Lusaka, the capital city of Zambia. The hospital is the principal eye care facility and the national referral center tending to between 13,000 and 14,000 patients annually from all over Zambia. Ethical approval for the study was obtained from the University of Zambia Biomedical Research Ethics Committee (UNZABREC IRB \# 015-05-17), Zambia National Health Research Authority, and the Institutional Review Board of the Uni- versity of Nebraska-Lincoln (IRB \# 20170817442FB). The study participants were consecutively sampled from among males and females aged 18 years and older scheduled for an excisional or incisional biopsy of OSTs suspicious for OSSN between November 2017 and November 2019.

Upon a decision by the ophthalmologist that a patient with an OST required surgical treatment, the recruitment nurse invited the patient to participate in the study. The counselor explained the goals of the study to the prospective participant, including procedures, information, and the tissue samples to be collected. Only patients who were freely willing to give informed consent, as indicated by a signature or thumbprint were recruited into this study. The exclusion criteria included any previous history of immunologic diseases such as lymphoma or unrelated cancer and a nondiagnostic histologic result.

Upon enrollment, an interviewer-administered broad-based questionnaire was used to collect data on sociodemographic factors, HIV status, duration of HIV positivity, prior CD-4 counts, and combined antiretroviral therapy use. The attending ophthalmologist took a comprehensive history, visual acuity, slit-lamp examination, and photographs of the affected eye. Following the interview, tears, blood, and buccal swab samples were collected. The excised tissue specimens obtained following the routine surgical management and tissues were bisected. One sample was fixed using $10 \%$ neutral buffered formalin for histopathologic analysis, and the other was immersed in RNAlater stabilization solution overnight and subsequently stored at $-80^{\circ} \mathrm{C}$ for molecular studies. Two hundred sixty-six (266) participants were recruited; 1 participant was excluded because of insufficient tissue for histologic diagnosis. All participants received an HIV serology test as part of the routine clinical care under the 'test, counsel, and treat' policy at UTH. CD4 count testing was done on the collected blood using Becton Dickinson's FACSCount dual platform system.

Clinical assessment included a description of lesion location, size, appearance, and extent of the primary tumor. We documented findings of any clinically palpable preauricular, submandibular, or cervical (regional) lymph nodes, and clinical evident distant metastasis.

Following a 24-48-h fixation, the biopsy tissue was grossed and embedded in paraffin wax blocks. Two, $5 \mu \mathrm{m}$ thick tissue sections were mounted on glass slides and stained with Mayer's Hematoxylin and Eosin, and mucicarmine and Hematoxylin stain, respectively. The tissue slides were previewed by a pathology resident for the adequacy of the section and preliminary diagnosis, and a senior pathologist determined the final histopathologic diagnosis. The presence or absence of koilocytosis and sun damage (solar elastosis) was determined. Reports for cancer cases included histologic type, category and grade, lymphovascular invasion, and perineural invasion.

Histologic classification of lesions was done according to the WHO classification of tumors of the eye, 4th ed. (2018) [2], following epithelial, melanocytic, hematolymphoid, soft tissue, hamartomas/choristomas, and secondary tumors. Epithelial tumors were subclassified as OSSN versus non-OSSN. Grading of preinvasive OSSN included conjunctiva-cornea intraepithelial neoplasia (CCIN I, CCIN II, and CCIN III) and carcinoma in situ. The categorization of invasive OSSN included histologic subtype and grade. The tumor grade categories included well-differentiated, moderately differentiated, poorly differentiated, and undifferentiated tumors. Tumor, node, metastasis classification for carcino- 


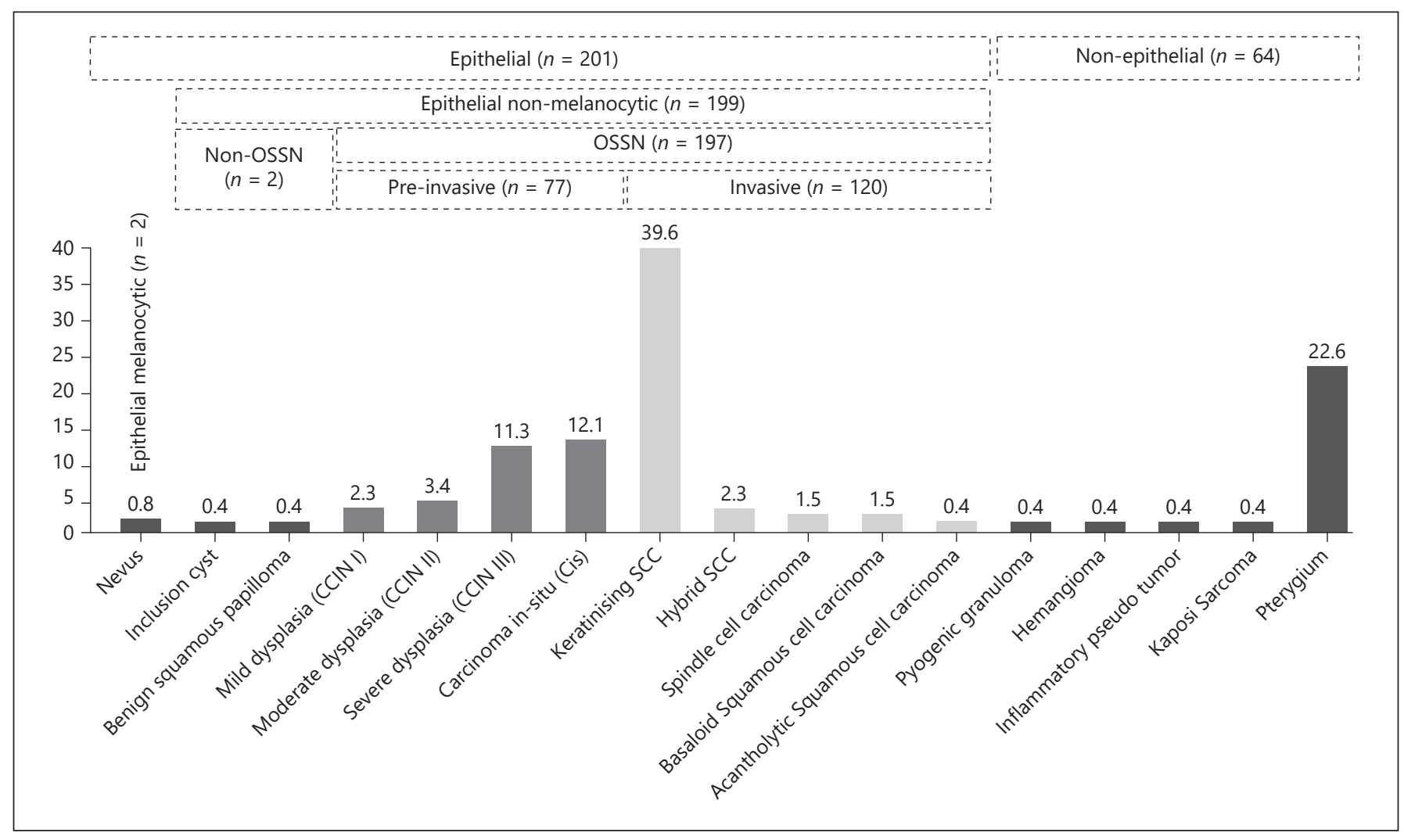

Fig. 1. Frequency of primary OSTs by origin and histopathology. OST, ocular surface tumor.

mas of the conjunctiva was done according to the 8th ed. of the American Joint Committee on Cancer staging manual (2018) [15] following clinical classification and microscopic confirmation of the tumor.

Data Management and Statistical Analysis

Participants were assigned a unique identification number upon recruitment in order to link questionnaires, medical chart reviews, clinical evaluations, and histology results. De-identified data were entered into 2 separate access files and stored in a secure cloud (Box application) restricted to the study team.

Access file records were compared using the dataset comparison feature on SPSS for data entry errors. IBM SPSS 25 statistical package was used for statistical analysis. The list of variables is included in online suppl. 1; for all online suppl. material, see www. karger.com/doi/10.1159/000511610.

These variables were grouped and presented as frequencies and percentages. Association was tested by $\chi^{2}$ (dichotomous) and bivariate correlations (nonparametric continuous variables vs. dichotomous variables; Kendall rank) at $p<0.05$. All assumptions for $\chi^{2}$ testing were observed. The strength of the association between the categorical variables was determined using phi, Cramer's V, and correlation coefficient. All assumptions for logistic regression were observed. The variable exclusion criteria included (i) $\chi^{2} /$ bivariate correlation (at a $p$ value $>0.2$ ) and variables with $20 \%$ of the expected frequency [16] and (ii) multicollinearity (tool: col- linearity diagnosis) was assessed using each dependent variable along with independent variables that passed the previous criteria. Variables with a variance inflation factor $>3$ were excluded. Binary logistics was used to adjust the odds ratios (ORs) for covariance. Adjusted OR, 95\% confidence interval (CI), and $p$ values were determined.

\section{Results}

Two hundred sixty-five participants were included in the final analysis. In Figure 1, histologic categories and specific diagnostic frequencies are shown including epithelial $(75.8 \%)$ and nonepithelial $(24.2 \%)$ tumors. Invasive OSSN lesions were the most frequent (45.3\%) OSTs seen, followed by preinvasive OSSN (29.1\%) and pterygium (22.6\%). Other lesions seen included 2 nevi $(0.8 \%)$ and 1 case each of Kaposi sarcoma, benign epithelial cyst, squamous papilloma, hemangioma, pyogenic granuloma, and inflammatory pseudotumor. Figures 2 and 3 show representative gross and histologic photos of specific tumor diagnoses, respectively. OSSN lesions includ- 

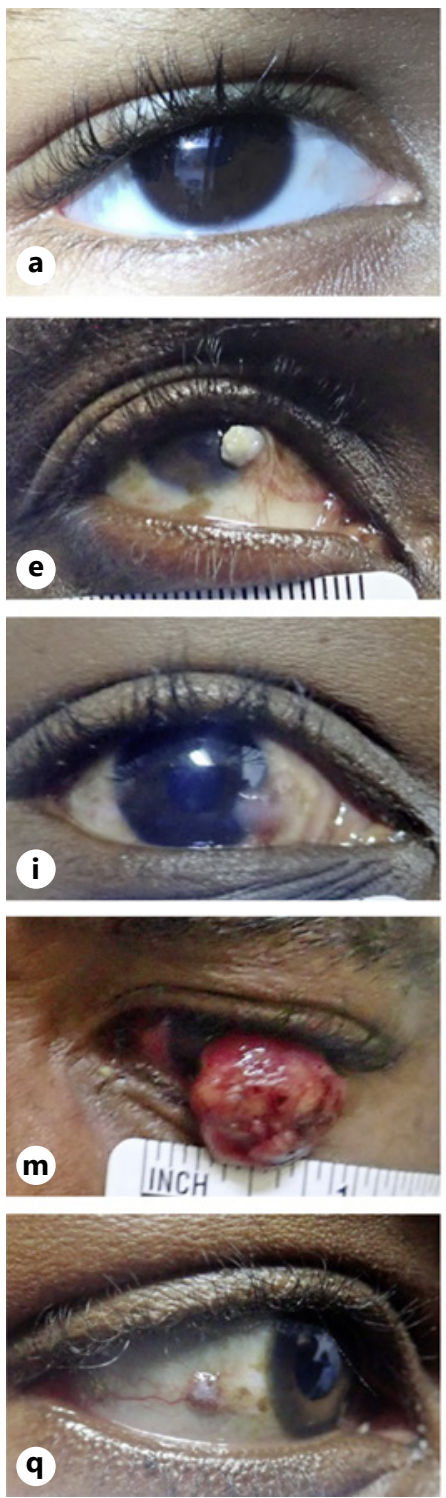
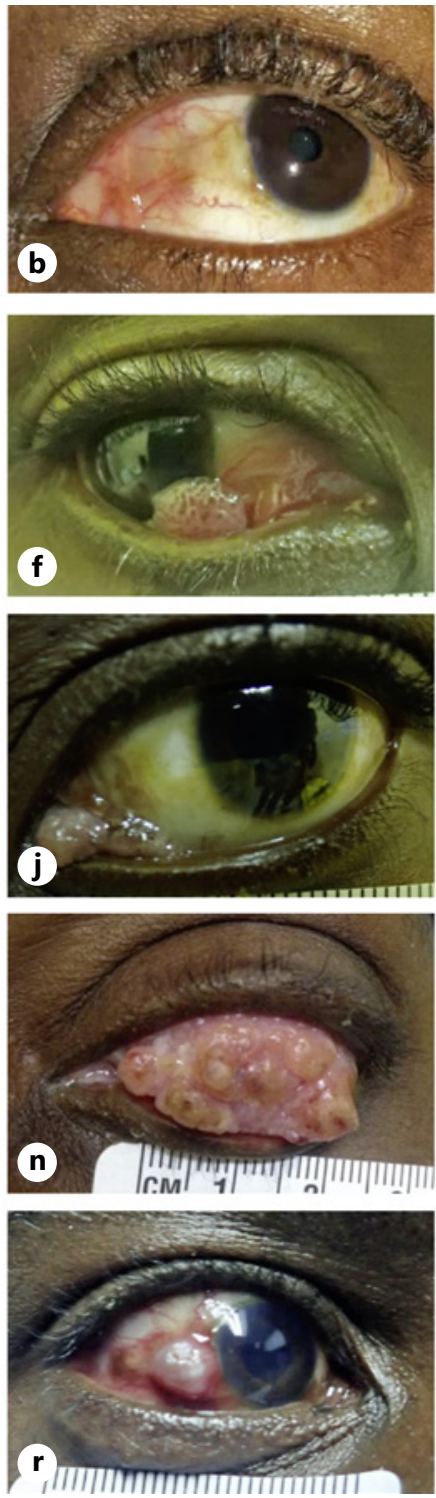
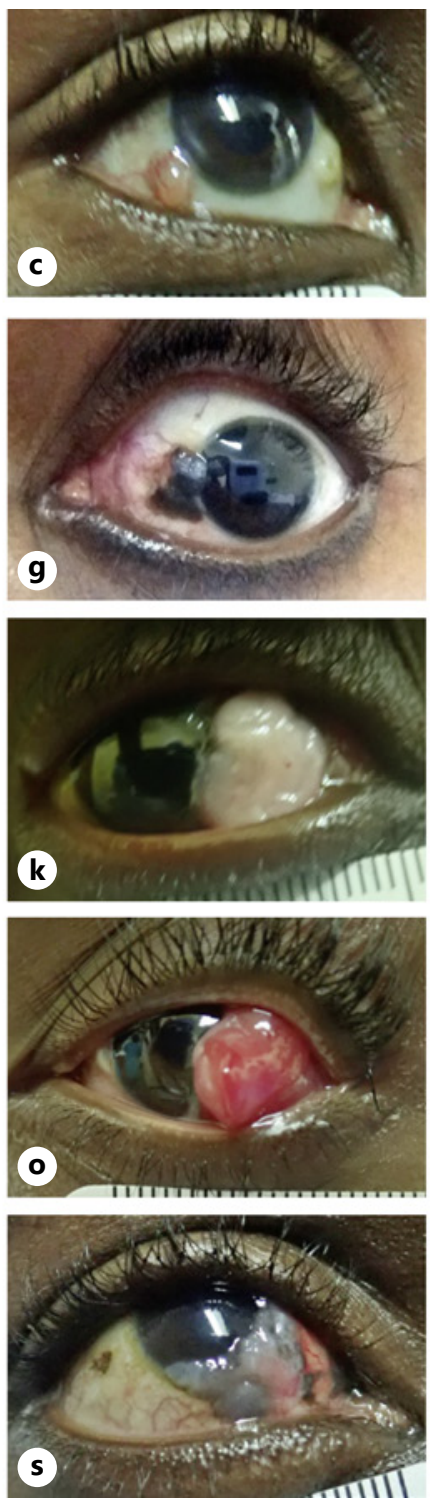
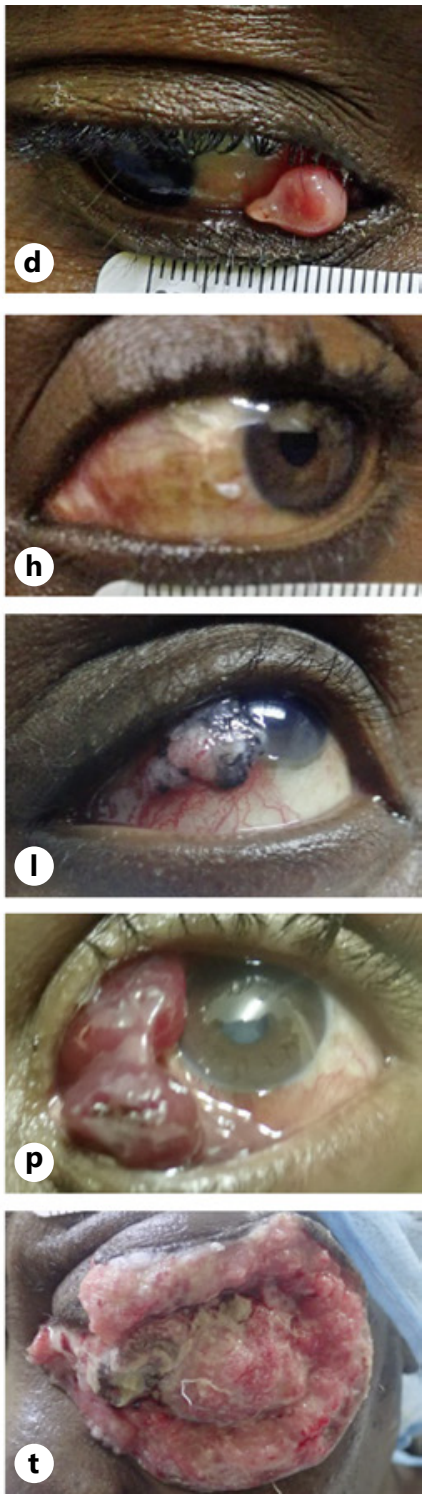

Fig. 2. Representative gross images of OSTs. Normal eye (a), pterygium with fibrovascular appearance $(\mathbf{b})$, conjunctiva epithelial inclusion cyst (c), pyogenic granuloma (d), benign squamous papilloma with leukoplakia (e), squamous papilloma with high-grade dysplasia (f), nevus with brown pigmentation (g), CIN - CIN II (h), CIN III (i), carcinoma in situ in a pedunculated lesion (j),

ed preinvasive $(39.1 \%)$ and invasive (60.9\%) lesions. CCIN-III and carcinoma in situ accounted for $80.5 \%$ of preinvasive OSSN. Invasive OSSN lesions included keratinizing (conventional) squamous cell carcinoma (KSCC) (87.5\%), basaloid SCC (3.3\%), spindle cell carcinoma (3.3\%), hybrid SCC (5.0\%), and acantholytic SCC (0.8\%). Most invasive tumors were moderately differentiated (74.2\%), while well-differentiated tumors were $14.2 \%$, minimal inflammation in SCC $(\mathbf{k})$, moderate inflammation in SCC (I), severe inflammation in pedunculated SCC $(\mathbf{m})$, basaloid SCC (n), spindle cell carcinoma (o), Kaposi Sarcoma (p). Representative images for primary tumor (T) stage - SCC (q-t). T1 (q), T2 $(\mathbf{r})$, T3 $(\mathbf{s})$, and T4 (t). OST, ocular surface tumor; SCC, squamous cell carcinoma. and poorly differentiated tumors were $11.6 \%$. Lymphovascular and perineural invasions were not observed in any of the invasive tumors. Koilocytosis was present in $18.6 \%$ of 242 cases assessed, while solar elastosis was present in $95.5 \%$ of 220 cases assessed. Of the 120 participants with invasive OSSN, 93.3\% presented late for the primary diagnosis (primary tumor stages T3 and T4). Clinical assessment revealed no signs or symptoms for distant tu- 

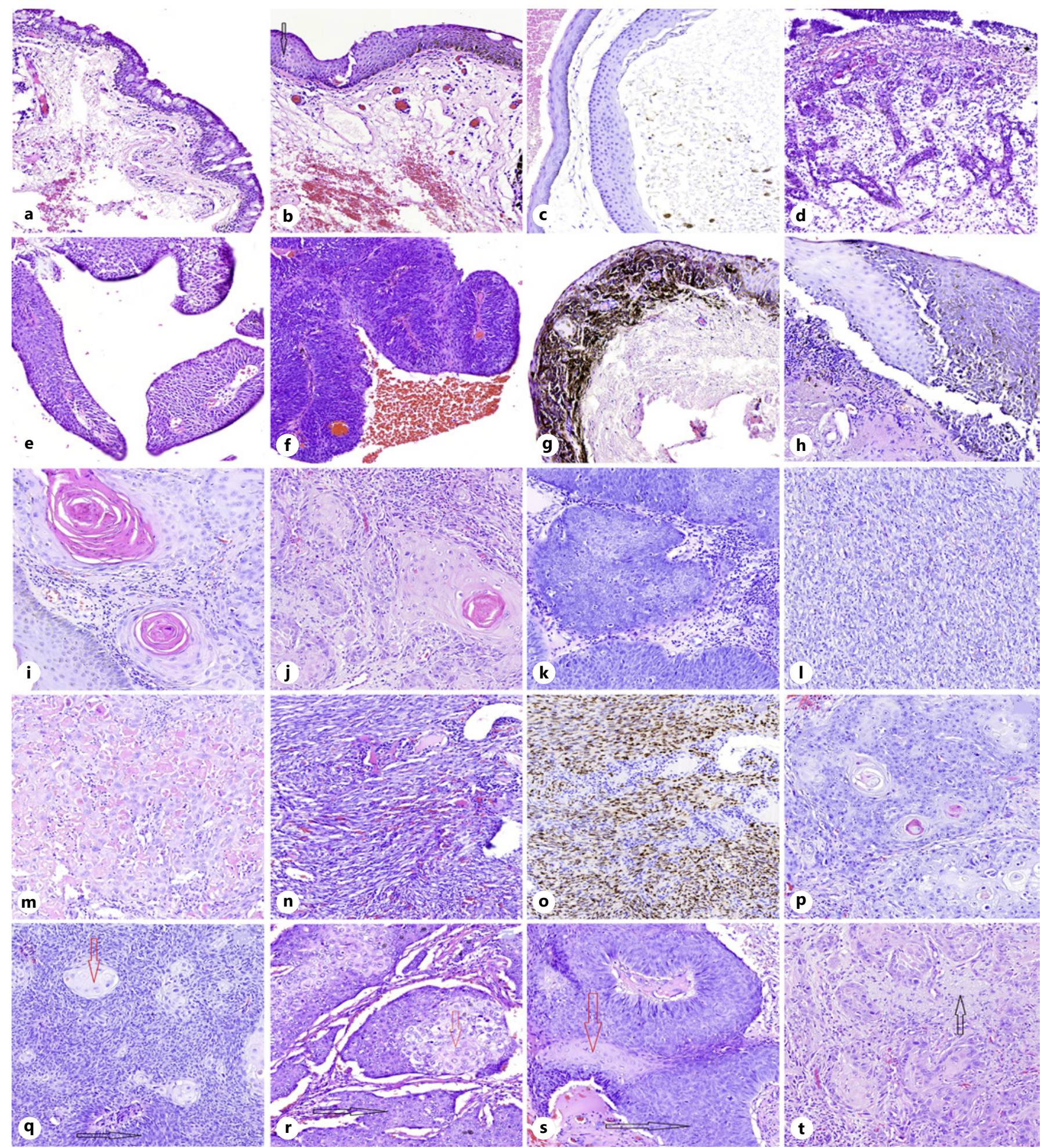

Fig. 3. Representative histology images of OSTs. Normal conjunctiva (a), pterygium with koilocytes (b), inclusion cyst (c), ulcer (d), conventional (keratinizing) SCC (i, j), basaloid SCC (k), spindle cell carcinoma (I), acantholytic SCC (m), Kaposi sarcoma with positive Lana immunohistochemistry $(\mathbf{n}, \mathbf{o})$, hybrid squamous car-

cinoma (p-s) and solar elastosis in well-differentiated SCC (t). Panels $\mathbf{a}$ through $\mathbf{n}$ and $\mathbf{p}$ through $\mathbf{t}$ are Hematoxylin-Eosin stained. Panel $\mathbf{o}$ is KSHV Lana immunohistochemistry. OST, ocular surface tumor; SCC, squamous cell carcinoma. 
Table 1. Comparison of clinical features of preinvasive OSSN, invasive OSSN, and combined OSSN with benign conjunctiva lesions on slit-lamp examination

\begin{tabular}{|c|c|c|c|c|c|c|c|c|c|c|}
\hline \multirow[t]{2}{*}{ Clinical features } & \multirow{2}{*}{$\begin{array}{l}\text { Benign lesions } \\
(n=67), n(\%)\end{array}$} & \multicolumn{3}{|c|}{ Preinvasive OSSN $(n=77)$} & \multicolumn{3}{|c|}{ Invasive OSSN $(n=120)$} & \multicolumn{3}{|c|}{ OSSN $(n=197)$} \\
\hline & & $n(\%)$ & $x^{2}$ & $\begin{array}{l}p \\
\text { value }\end{array}$ & $n(\%)$ & $\chi^{2}$ & $\begin{array}{l}p \\
\text { value }\end{array}$ & $n(\%)$ & $x^{2}$ & $\begin{array}{l}p \\
\text { value }\end{array}$ \\
\hline \multicolumn{11}{|l|}{ Location of the lesion (mostly) } \\
\hline Nasal limbus & $56(83.6)$ & $54(70.1)$ & 3.595 & 0.058 & $81(67.5)$ & 5.677 & 0.017 & $135(70.7)$ & 5.664 & 0.017 \\
\hline Temporal limbus & $8(11.9)$ & $18(23.4)$ & 3.167 & 0.075 & $45(37.5)$ & 13.830 & 0.0001 & $63(32)$ & 10.212 & 0.001 \\
\hline Superior limbus & $5(7.5)$ & $3(3.9)$ & 0.869 & 0.351 & $33(27.5)$ & 10.661 & 0.001 & $36(18.3)$ & 4.455 & 0.035 \\
\hline Inferior limbus & $6(9)$ & $4(5.2)$ & 0.784 & $0.288^{*}$ & $38(31.7)$ & 12.325 & 0.0001 & $42(21.3)$ & 5.138 & 0.023 \\
\hline Circumlimbal & $1(1.5)$ & $1(1.3)$ & 0.01 & 0.921 & $22(18.3)$ & 11.304 & 0.001 & $23(11.7)$ & 6.272 & 0.012 \\
\hline Corneal & $7(10.4)$ & $9(11.7)$ & 0.056 & 0.813 & $33(27.5)$ & 7.435 & 0.006 & $42(21.3)$ & 3.910 & 0.048 \\
\hline Caruncle & $5(7.5)$ & $3(3.9)$ & 0.869 & 0.351 & $22(18.3)$ & 4.113 & 0.043 & $25(12.7)$ & 1.357 & 0.244 \\
\hline Bulbar conjunctiva & $39(58.2)$ & $42(54.5)$ & 0.195 & 0.658 & $65(54.2)$ & 0.285 & 0.594 & $107(54.3)$ & 0.307 & 0.580 \\
\hline Inferior fornix & $1(1.5)$ & $2(2.6)$ & 0.214 & $0.552^{*}$ & $5(4.2)$ & 0.990 & $0.300^{*}$ & $7(3.6)$ & 0.723 & $0.354^{*}$ \\
\hline Nasolacrimal system & $1(0.9)$ & $1(1.3)$ & 0.01 & 0.716 & $10(5.9)$ & 3.634 & $0.049^{*}$ & $11(5.6)$ & 1.929 & $0.145^{*}$ \\
\hline Forniceal palpebra & $2(3)$ & $3(3.9)$ & 0.089 & 0.766 & $26(21.7)$ & 11.786 & 0.001 & $29(14.7)$ & 6.644 & 0.010 \\
\hline Pretarsal conjunctiva & $0(0)$ & $0(0)$ & na & na & $7(5.8)$ & 4.060 & $0.042^{*}$ & $7(3.6)$ & 2.446 & 0.125 \\
\hline Eyelid & $0(0)$ & $1(1.3)$ & 0.876 & $0.535^{*}$ & $4(3.3)$ & 2.282 & $0.167^{*}$ & $5(2.5)$ & 1.733 & $0.228^{*}$ \\
\hline \multicolumn{11}{|l|}{ Lesion appearance } \\
\hline Leukoplakia & $31(46.3)$ & $42(54.5)$ & 0.982 & 0.322 & $47(39.2)$ & 0.892 & 0.345 & $89(45.2)$ & 0.024 & 0.877 \\
\hline Erythroplakia & $4(6)$ & $6(7.8)$ & 0.184 & 0.668 & $13(10.8)$ & 1.230 & 0.267 & $19(9.6)$ & 0.849 & 0.357 \\
\hline Gelatinous appearance & $5(7.5)$ & $6(7.8)$ & 0.006 & 0.941 & $16(13.3)$ & 1.486 & 0.223 & $22(11.2)$ & 0.747 & 0.387 \\
\hline Fibrovascular appearance & $20(29.9)$ & $5(6.5)$ & 13.623 & 0.0001 & $7(5.8)$ & 20.075 & 0.0001 & $12(6.1)$ & 26.496 & 0.0001 \\
\hline Papilliform appearance & $1(1.5)$ & $3(3.9)$ & 0.766 & $0.364^{*}$ & $10(8.3)$ & 3.634 & $0.049^{*}$ & $13(6.6)$ & 2.596 & $0.090^{*}$ \\
\hline Brown lesion pigment & $5(7.5)$ & $15(19.5)$ & 4.326 & 0.038 & $6(5.0)$ & 0.471 & $0.350^{*}$ & $21(10.7)$ & 0.576 & 0.448 \\
\hline Fungating & $1(1.5)$ & $0(0)$ & na & na & $21(17.5)$ & 10.613 & 0.001 & $21(10.7)$ & 5.500 & 0.019 \\
\hline \multicolumn{11}{|l|}{ Other features } \\
\hline Lesion feeder vessels & $38(56.7)$ & $43(55.8)$ & 0.011 & 0.916 & $89(74.2)$ & 6.008 & 0.014 & $132(67)$ & 2.308 & 0.129 \\
\hline Corneal involvement & $21(31.3)$ & $29(37.7)$ & 0.631 & 0.427 & $56(46.7)$ & 4.168 & 0.041 & $85(43.1)$ & 2.899 & 0.089 \\
\hline Adhesion to underlying sclera & $4(6)$ & $4(5.2)$ & 0.041 & 0.561 & $26(21.7)$ & 7.865 & 0.005 & $30(15.2)$ & 3.819 & $0.051 S$ \\
\hline Widest diameter, mm, median (IQR) & $6(6-9)$ & $6(5-8)$ & -0.024 & 0.742 & $5(5-8)$ & -0.029 & 0.656 & $5.2(5-7)$ & -0.024 & 0.657 \\
\hline \multicolumn{11}{|c|}{ Inflammation } \\
\hline None & $29(44.6)$ & $27(36)$ & 3.059 & 0.368 & $28(23.5)$ & 13.042 & 0.005 & $55(28.4)$ & 9.571 & 0.023 \\
\hline Minimal & $27(41.5)$ & $29(38.7)$ & & & $78(40.2)$ & & & & & \\
\hline Mild & $5(7.7)$ & $9(12)$ & & & $34(17.5)$ & & & & & \\
\hline Moderate/severe & $4(6.2)$ & $10(13.3)$ & & & $27(13.9)$ & & & & & \\
\hline \multicolumn{11}{|c|}{ Distance vision impairment affected eye by visual acuity (VA) } \\
\hline Normal - VA 6/12 or better & $52(77.6)$ & $60(77.9)$ & 2.020 & 0.732 & $65(55.1)$ & 14.385 & 0.006 & $125(64.1)$ & 8.392 & 0.078 \\
\hline Mild - VA worse than 6/12 & $3(4.5)$ & $2(2.6)$ & & & $7(3.6)$ & & & & & \\
\hline Moderate - VA worse than 6/18 & $7(10.4)$ & $7(9.1)$ & & & $19(9.7)$ & & & & & \\
\hline Severe - VA worse than $6 / 60$ & $2(3.0)$ & $1(1.3)$ & & & $7(3.6)$ & & & & & \\
\hline Blindness - VA worse than $3 / 60$ & $3(4.5)$ & $7(9.1)$ & & & $37(19.0)$ & & & & & \\
\hline
\end{tabular}

OSSN, ocular surface squamous neoplasia. * Indicates Fisher's exact test was used.

mor metastasis in any participant. Enlarged regional lymph nodes were clinically palpable in 6 patients $(2.3 \%)$, all with invasive OSSN and at an advanced tumor stage T3/T4; however, none of these were biopsied for histologic assessment for the presence of a tumor. Participants with nonconventional SCC all had advanced stage T3/T4 disease. The benign non-OSSN cases were grouped and compared with preinvasive and invasive OSSN to deter- mine possible associations. The participant with a histologic diagnosis of Kaposi sarcoma was not included in the analysis for an OSSN association.

\section{Demographic Characteristics}

Table 1 summarizes the demographic characteristics of 264 participants grouped based on tumor category; OSSN (preinvasive, invasive, and combined) versus be- 
nign ocular surface lesions. The median age of the participants was 36 (IQR: 33-41) years, with no significant difference between patients with OSSN and benign lesions. The male: female ratio was 2:3 for all participants, with no significant difference between patients with OSSN versus benign lesions. Participants diagnosed with preinvasive OSSN were slightly younger than those with invasive OSSN (38 vs. 41 years). Most participants were married (60\%), reached secondary school (49.8\%), and were in formal or informal employment (74.5\%). Marital status, level of education, employment status, and household income were similar in the disease categories. Any smoking and alcohol consumption history were reported in 17 and $43.3 \%$ of the participants, respectively, and were associated with a probable moderate $(p=0.04$, phi: 0.150 $[p=0.04])$ and strong $(p=0.03$, phi: $0.159[p=0.03])$ risk for invasive OSSN, respectively.

\section{Clinical Features: Symptoms}

Online suppl. Table 2 (in online suppl. 1) summarizes the primary symptoms at the time of presentation and compares participants with preinvasive and invasive OSSN with benign lesions. The most frequent symptoms included growth (84.5\%), pain (65.3\%), foreign body sensation (64.9\%), redness (56.2\%), and a white spot on the eye $(47.5 \%)$. All the presenting symptoms were present in all disease categories and were generally similar between preinvasive OSSN and benign lesions. However, excessive tearing $(p=0.017)$ and a white spot on the eye $(p=$ 0.011 ) were strongly associated with preinvasive OSSN. Participants with a diagnosis of invasive OSSN were more likely to complain of pain $(p=0.007)$, redness $(p=0.034)$, excessive tearing $(p=0.0001)$, discharge $(p=0.011)$, a history of bleeding from the lesion $(p=0.007)$ and reduced vision ( $p=0.0001)$ in the affected eye. A history of prior herbal medicine use was strongly associated with preinvasive OSSN ( $p=0.043$ ), but not invasive OSSN ( $p=$ $0.516)$; however, its use was in a small proportion $(8.7 \%)$ of the participants. There was no significant difference in the duration of symptoms from onset to diagnosis, prior surgical or medical treatment history, itchiness or foreign body sensation, and prior lesion or trauma in the affected eye between patients with OSSN and benign lesions.

\section{Clinical Features: Signs}

Table 1 shows the examination findings. The right eye was the most frequently affected (55.5\%). Two patients had bilateral lesions; however, a biopsy was only taken from one eye at recruitment. Both OSSN and benign lesions were noted to involve the nasal, temporal, superior, and inferior limbus, as well as the cornea and caruncle. None of the benign lesions extended from the bulbar conjunctiva to the pretarsal conjunctiva or the eyelid. Most tumors arose from the nasal limbus (72.1\%) and the temporal limbus (26.4\%). Tumor extension to the cornea was present in $60.5 \%$ of the participants. There was no difference in the location of the tumor between participants with preinvasive OSSN and benign lesions. Benign lesions were more likely to be at the nasal limbus $(p=0.017)$ compared to invasive OSSN. Invasive OSSN lesions, when compared to benign lesions, were more likely to be at the temporal limbus $(p=0.0001)$ and to involve the inferior limbus $(p=0.0001)$, superior limbus $(p=0.001)$, circumlimbal $(p=0.001)$ and to extend from the bulbar conjunctiva to the cornea $(p=0.006)$, the caruncle $(p=$ $0.043)$, nasolacrimal system $(p=0.049)$, forniceal palpebral $(p=0.001)$, and the pretarsal conjunctiva (0.042). There was no difference between the location of the lesions on the bulbar conjunctiva ( $p=0.594)$ between participants with invasive OSSN and benign lesions. Preinvasive OSSN ( $p=0.008)$ and invasive OSSN lesions ( $p=$ 0.0001 ) were significantly larger than benign lesions.

All the varied lesion appearances and the graded levels of inflammation were present in benign and OSSN lesions. A fibrovascular appearance was strongly associated with benign lesions ( $p=0.010)$, while a brown lesion appearance was associated with preinvasive OSSN lesions. A fungating lesion $(p=0.001)$ and a papilliform appearance $(p=0.049)$ were more frequently seen in invasive OSSN lesions.

Leukoplakia, erythroplakia, and a gelatinous appearance occurred at a similar frequency in the varied lesions. Absent inflammation was seen more frequently in benign lesions, while moderate to severe inflammation was seen with invasive OSSN $(p=0.023)$. Feeder vessels and adhesion to the underlying stroma were seen with benign and OSSN lesions; however, their presence was strongly associated with invasive OSSN. Forty-six (15.1\%) of the participants were presented with blindness in the affected eye by the WHO standards. Severe and moderate distance vision impairment was observed in 3.4 and $9.8 \%$ of the participants. Blindness in the affected eye was associated with invasive OSSN $(p=0.005)$.

\section{HIV Infection and Ocular Surface Tumors}

Figure 4 shows the HIV/ART history. HIV prevalence in the participants was $68.3 \%$ and was significantly higher in patients with OSSN (74.6\%) versus benign lesions $(49.3 \%)(p=0.0001)$. The majority of participants $(92.5 \%)$ reported that they had been tested for HIV before. Among 

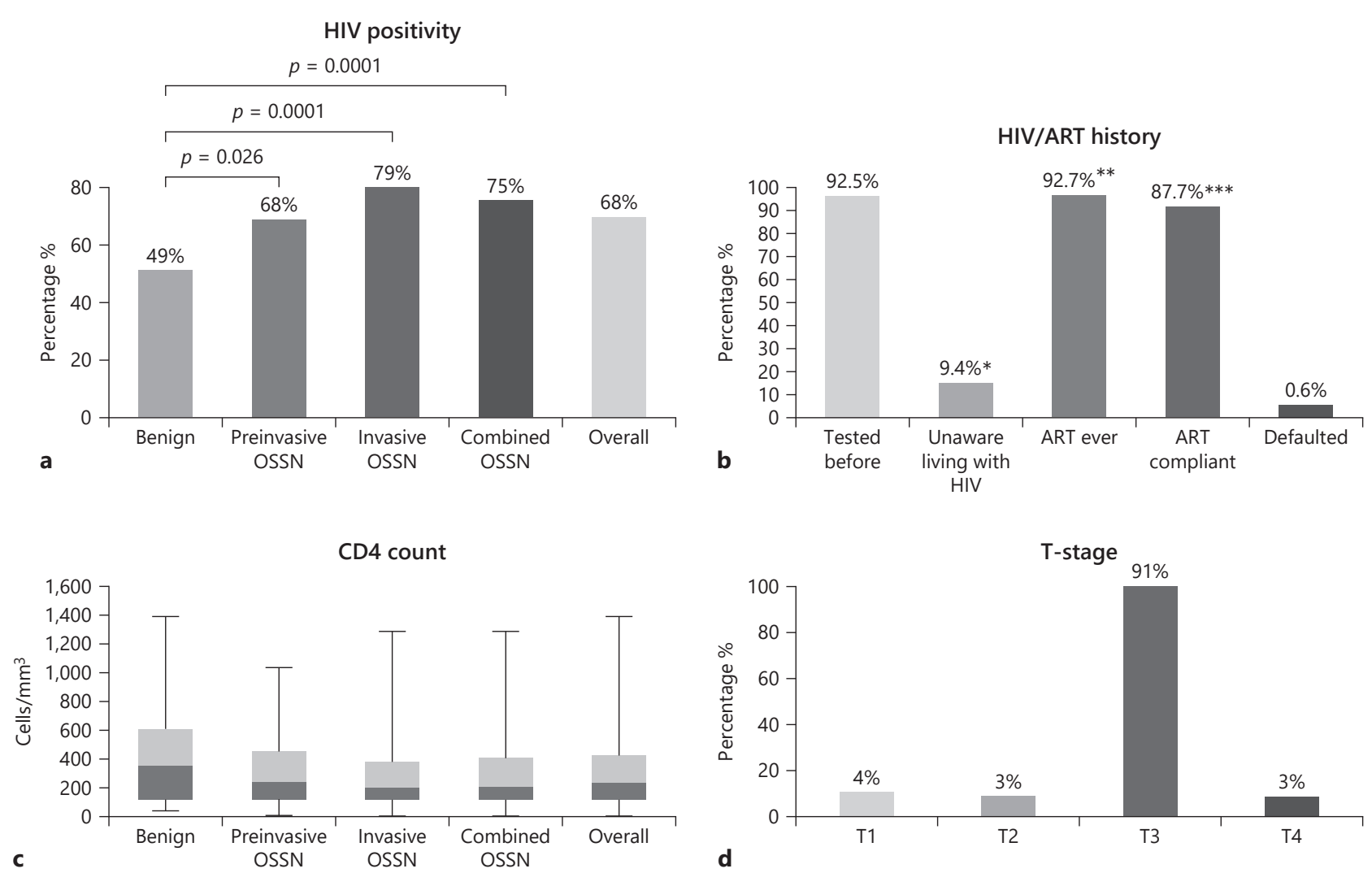

Fig. 4. HIV positivity (a), HIV/ART history (b), median CD4+ counts (c), and primary tumor (T) stage of invasive cancer $(\mathbf{d})$.

the HIV positive participants, 17 (9.4\%) were not aware that they were living with HIV at the time of presentation. Of the 164 participants that knew that they were HIV positive before enrollment into the study, $92.7 \%$ reported that they were taking highly active antiretroviral therapy (HAART), and $87.7 \%$ were strictly compliant with taking their medication. One patient reported that they had stopped taking their medicines. The history of HAART uptake was similar between participants with OSSN and benign lesions. The median duration of HAART was preinvasive OSSN 7 (IQR: 1-60) months, invasive OSSN 16 (IQR: 12-24) months, combined OSSN 12 (IQR: 12-24) months, and benign lesions 24 (IQR: 1-60) months.

The median duration from the time of HIV diagnosis to starting HAART was benign 0.25 (0.25-9) months, preinvasive: 0.25 (IQR: $0.25-3$ ) months, invasive 0.25 (IQR: $0.25-1$ ) months, and combined OSSN 0.25 (IQR: $0.25-1)$ months. At recruitment, 165 (91.2\%) of the HIV positive participants had their CD4 count checked. The median CD4 count overall was 235 (IQR: 117-1,383) cells $/ \mathrm{mm}^{3}$, and $78.2 \%$ of the CD4 counts were below 500 cells $/ \mathrm{mm}^{3}$ (Fig. 4). The median CD4 count in patients with benign lesions was $356 \mathrm{cells} / \mathrm{mm}^{3}$, and in preinvasive OSSN, the CD4 count was 238 cells $/ \mathrm{mm}^{3}$, and in invasive OSSN, the CD 4 counts were 200 cells $/ \mathrm{mm}^{3}$. However, there was no significant difference in the CD4 count between the groups $(p=0.095)$. The prevalence of HIV in patients with invasive OSSN was $79.2 \%$. Of the $25 \mathrm{HIV}$ negative participants with invasive OSSN, $88 \%$ had advanced disease stage (T3/T4) at diagnosis.

\section{Multivariate OSSN Associations}

Benign versus preinvasive OSSN regression analysis (Table 2, online suppl. Table 3 [in online suppl. 1]) revealed associations with HIV positivity (adjusted OR [aOR] 2.332, 95\% CI 1.03-5.29, $p=0.043$ ); appearance of red eye $(3.147,1.3-7.55, p=0.010)$; appearance of teary 
Table 2. Analysis of predictors for preinvasive OSSN, invasive OSSN, and combined OSSN

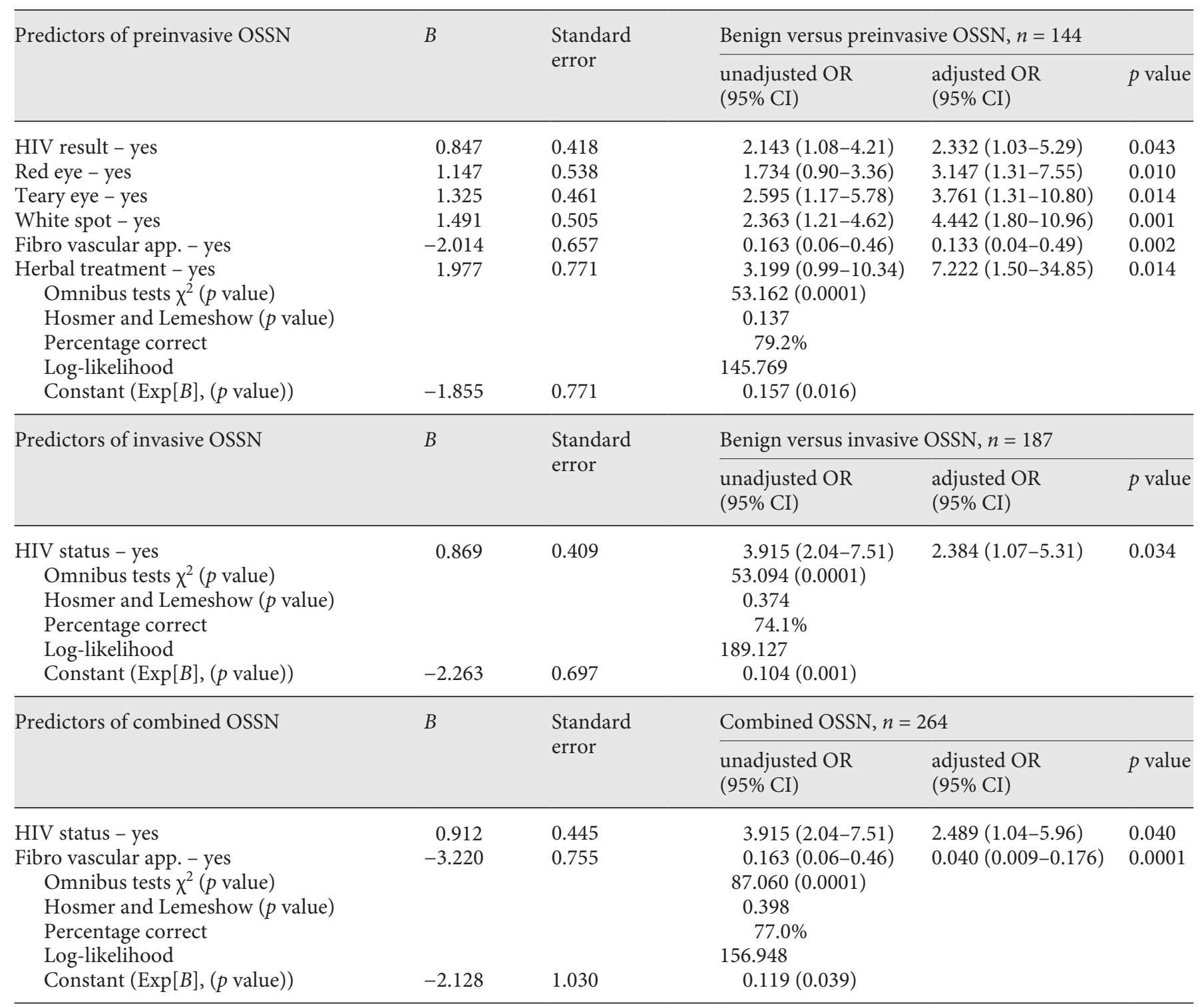

OSSN, ocular surface squamous neoplasia.

eye $(3.761,1.31-10.80, p=0.014)$; presence of white spot in affected eye $(4.442,1.80-10.96, p=0.014)$; absence of fibrovasculature $(0.133,0.04-0.49, p=0.002)$; and prior use of herbal treatment $(7.222,1.50-34.85, p=0.014)$. Benign versus invasive lesions had adjusted associations with HIV positivity $(2.384,1.07-5.31, p=0.034)$ alone. Benign versus OSSN found associations after adjustment with HIV positivity $(2.489,1.04-5.96, p=0.04)$, appearance of red eye $(2.957,1.23-7.13,0.016)$, and the absence of fibrovasculature $(0.040,0.0009-0.176, p=0.0001)$.
HIV positivity was a common predictor between the dependent variables and presence of OSSN associated with the absence of fibrovasculature.

\section{Discussion}

Histopathologic evaluation of OSTs is essential since accurate information about regional disease arrays and their distinguishing clinical features is required when de- 
signing programs that promote strategies for prevention and access to care, early detection, diagnosis, treatment, and palliation and for a better understanding of the natural history of a disease. In our study, epithelial nonmelanocytic lesions (predominated by OSSN) comprised the most common tumor category, similar to other studies from Africa [3, 7], in contrast to studies from temperate climates where epithelial-melanocytic (mostly benign) tumors predominate $[9,17,18]$. This agrees with previous findings that melanocytic tumors of the ocular surface are rare in black populations [19]. OSSN predominantly consisted of invasive lesions, while preinvasive OSSN lesions were predominant in other studies $[2,3,9,11]$. This finding is probably due to the clinician's decision only to biopsy malignant looking lesions. Pterygium, the most frequently seen tumor in primary care settings $[2,20]$ was the third most frequent lesion seen in our study after preinvasive OSSN. This can be attributed to the fact that, in the early stages, pterygia are treated conservatively through close follow-up [20]. While a benign squamous papilloma is said to be common [21], we saw only one case. Most of the papillomatous lesions seen at clinical evaluation turned out to have varying degrees of dysplasia with some displaying overt stromal invasion; hence, these were classified under the OSSN category. Despite the high HIV seropositivity rates reported in our study, we only saw only one Kaposi sarcoma of the ocular surface. Few case studies have reported Kaposi sarcoma of the conjunctiva [22-24].

OSSN incidence has two broad patterns and includes one seen in immunosuppressed populations from subSaharan Africa $[2,3,5]$ and the other in immunocompetent populations in temperate countries $[25,26]$. The disease patterns seen in our study followed that which has been observed in other sub-Saharan Africa studies. However, our patients consisted mostly of young people and women, both of whom were HIV positive. This is in contrast to findings in more temperate climates where OSSN lesions are the second most common OSTs after melanocytic tumors, and it occurs predominantly occurs in elderly males with increasing incidence associated with age, sunlight exposure, and fairer skin $[2,5,9]$.

While invasive OSSN is rare and occurs predominantly in males globally $[2,26,27]$, it was the most frequently seen OSTs in our study and occurred most frequently in females with more than $80 \%$ of the patients below the age of 50 years similar to studies from Africa [25]. The increased frequency of invasive OSSN among young adults in sub-Saharan Africa is due to the high prevalence of HIV and risk for infection $[5,28]$. There was no difference seen

Clinical and Pathologic Presentation of Primary Ocular Surface Tumors with sex, mean age, marital status, level of education, employment status, and household income between patients with OSSN and benign lesions. It is expected that higher education, a social support system, and higher household income may lead to earlier health-seeking behavior. In Kenya, Gichuhi et al. [3] found that a lower education level and being widowed was associated with OSSN.

KSCC was the most frequent variant of invasive OSSN, as described previously $[2,28]$. KSCC has a favorable prognosis with rare metastasis [29]. Other variants of invasive SCC that we saw included spindle cell carcinoma and basaloid SCC. Unusual carcinomas of the conjunctiva included hybrid SCC and acantholytic SCC, and to our knowledge, these have not been previously described.

Diagnosis of spindle cell carcinoma was based on the microscopic predominance of malignant spindle cells. Spindle cell carcinomas are rare [2] and their incidence peak in the fifth to seventh decades with no sex preference [25], in contrast to our findings where all patients were female, HIV positive, and had a mean age of $35.0 \pm 6.6$ (ranges 29-42) years. Conjunctiva spindle cell carcinoma is classified because of its rarity, spindle histomorphology, epithelial and myoepithelial differentiation, unfavorable prognosis (increased tendency toward local recurrence, local invasion, and metastasis), and lack of standardized treatment $[2,30]$. We were unable to demonstrate the epithelial-mesenchymal transition in the three cases due to the unavailability of immunohistochemistry at our center.

Acantholytic SCC is a rare histologic variant of SCC that is characterized by acantholysis and dyskeratosis of the tumor cells due to loss of desmosome adhesion proteins [31-33]. It is classically seen in sun-exposed areas such as the skin and lip though other rare sites are reported, and it may have a poorer prognosis [31-33]. Hybrid SCC is characterized by focal partial squamous maturation (mature squamous cells with eosinophilic cytoplasm and distinct cell borders) in an otherwise poorly differentiated tumor, comprised of cellular areas with hyperchromatic nuclei and high nuclear: cytoplasm ratio [34, 35]. Hybrid SCC is well described in tumors of the oropharynx and is associated with human papillomavirus (HPV) infection [34-36]. To our knowledge, this is the first case of acantholytic SCC, and the first cases of hybrid SCC arising from the ocular surface reported.

The majority of our participants had advanced-stage disease at diagnosis, similar to reports from studies from sub-Saharan Africa [5, 37, 38]. The SCC variants all had primary tumor stage III/IV disease at presentation. The finding of advanced tumor stage even among the HIV seronegative patients implies that late presentation among 
OSSN patients in this region may be due to other reasons aside from HIV infection, and this needs investigation. None of the participants had clinical features suggestive of distant metastasis consistent with previous findings that the disease is slow-growing [29]. Only 6 of the 120 patients with invasive OSSN had enlarged (palpable) regional lymph nodes, in keeping with other studies $[3,29$, 30]. We have not reported margin status because we could not adequately assess tissue margins for a tumor because, upon excision, tissue was bisected and submitted for molecular studies and histology without orientation for the pathologist. Patients with preinvasive and early invasive tumor stages, (T1/T2) OSSN, were treated with Mitomycin C, while patients with high-stage (T3/T4) disease were referred to the cancer disease hospital for further management. The majority of the conventional SCCs were moderately differentiated, just like in other studies $[3,39]$.

\section{Clinical Features}

OSTs shared symptoms regardless of whether they were OSSN or benign lesions like in other studies $[3,5]$. The most frequent symptoms were growth on the eye, redness, and irritation, and the frequency of the symptoms was similar between patients with preinvasive and benign lesions. However, most symptoms were more frequent in patients with invasive OSSN lesions. The median duration of symptoms from onset to diagnosis was similar in the variable groups and averaged 7 months at presentation. Previous studies from Africa showed a similar trend of delayed presentation of patients with OSTs [5, $37,38]$. While some symptoms were strongly associated with invasive OSSN lesions, none of them was exclusive to a particular disease category, and hence, there is still a need for strengthening of pathology services in sub-Saharan countries to aid in definitive diagnosis $[3,5]$.

Most OSTs involved the nasal limbus and were unilateral just like previously seen [5]. Most benign lesions were likely to arise from the nasal limbus like OSSN, but a location other than the nasal limbus increased the likelihood for OSSN. Invasive OSSN was significantly larger than benign lesions, as previously shown [3]. The local invasion was seen across the lesion categories through corneal involvement and adhesion to the underlying sclera. Pterygia though considered as degenerative lesions with no malignant potential $[20,40]$ share similar features with OSSN lesions with regard to site of development, clinical features, etiologic factors, and propensity to invade with high recurrence rates following excision [41, 42]. Unlike preinvasive lesions, invasive OSSN and ad- vanced lesions were easily distinguished from benign lesions by size and a greater tendency to extend to the adjacent structures. Preinvasive and early invasive OSSN lesions shared overlapping clinical features with benign lesions which made clinical distinction difficult; a finding showed previously $[3,30]$.

The etiopathology of OSSN lesions is not yet fully understood; currently, multiple factors are thought to be associated with its development [5]. In our study, any smoking and alcohol consumption were associated with invasive OSSN, but not preinvasive OSSN or combined OSSN lesions. The role of smoking as a risk factor for OSSN etiology is still controversial as studies have shown variable results $[3,5]$. HPV cytopathic effects (Koilocytosis) infrequently occurred in OSTs in our study, and it was not associated with OSSN lesions. The role of HPV in OSSN etiology is still inconclusive with a systematic review by Carreira concluding that only cutaneous HPV may play a role [43]. HIV infection frequently occurred across the spectrum of OSTs, and it was strongly associated with OSSN, similar to findings from the region [44]. Solar elastosis was present across the varied spectrum of OSTs in keeping with previous findings [45]; however, bilateral disease was rare despite the attributed causal role of ultraviolet radiation $[3,5,30,45]$. CD4 count and ART uptake were not associated with the development of OSSN lesions. Because the majority of the HIV-positive participants were taking ART consistently, and ART uptake or CD4 count was not associated with the development of any of the OSTs, this is in agreement with other studies that reported that OSSN lesions could occur at any time of the HIV disease course $[46,47]$. This strengthens the view that ART uptake and CD4 cell count in the HIV infected host or those repopulated after ART may be dysfunctional and ineffective for immune surveillance of malignant cells $[5,48]$. However, we do not know whether OSSN developed before or after the commencement of ART, and the extent of the immune damage (how low $\mathrm{CD} 4$ counts decreased) during the disease course, and whether there is a preferential CD4 cell lineage that is damaged to explain ineffective immune surveillance for malignant cells.

OSSN, predominated by invasive carcinoma, was the most frequently found lesion in biopsied OSTs in the 265 adult patients in the present study. OSTs rarely presented as bilateral disease. Patients were relatively young, mostly female, and HIV positive. OSTs occurred regardless of ART uptake and CD4 count status. KSCC constituted 92.5\% of invasive carcinomas. Unusual invasive carcinomas seen included 6-hybrid SCC and 1 acantholytic SCC. Invasive carcinomas were mostly (94\%) at an advanced 
disease stage (T3/T4) at diagnosis. Risk factors for OSSN were smoking, alcohol use, and HIV infection. Preinvasive and invasive OSSN shared overlapping clinical features with benign lesions which made a distinction based on clinical impression alone very difficult. For this reason, diagnostic pathology services in low- and middle-income country hospitals need strengthening and modernization.

\section{Acknowledgements}

We thank members of the Angeletti and Wood laboratories for a critical discussion of this work.

\section{Statement of Ethics}

This study was performed with the highest ethical standards per the World Medical Association Declaration of Helsinki. The subjects provided their written informed consent to join the study, which included photo documentation and biopsies of their ocular tumors. The procedures and study protocol were approved by the University of Zambia Teach Hospital Institutional Review Board (UNZABREC IRB \# 015-05-17).

\section{Conflict of Interest Statement}

The authors have no competing interests.

\section{Funding Sources}

This project is part of the Zambia AIDS Malignancies Diagnosis and Pathogenesis Program (ZAMDAPP), which was funded entirely by the National Institutes of Health (U54CA221204).

\section{Author Contributions}

P.J. managed the research study at the UTH, provided pathology expertise, and wrote the first draft of the manuscript. S.N.S. performed the statistical analysis; P.M. performed all participant recruitment and ophthalmic procedures. F.M. and T.K. assisted with pathology work and helped write parts of the manuscript. G.K. helped perform microscopy. J.T.W. provided onsite laboratory support logistics in Zambia, C.W. provided logistics and study advice, and P.C.A. conceived of the study, analyzed the results, and helped write and edit the manuscript.

\section{References}

1 Zimmermann-Paiz MA, de la Riva JCG. Conjunctival tumors in children: histopathologic diagnosis in 165 cases. Arq Bras Oftalmol. 2015;78(6):337-9.

2 Grossniklaus HE, Eberhart C, Kivela T. WHO classification of tumours of the eye (medicine). 4th ed. 2018. p. 17-9.

3 Gichuhi S, Macharia E, Kabiru J, Zindamoyen $\mathrm{AM}$, Rono $\mathrm{H}$, Ollando E, et al. Clinical presentation of ocular surface squamous neoplasia in Kenya. JAMA Ophthalmol. 2015 Nov; 133(11):1305-13.

4 Honavar SG, Manjandavida FP. Tumors of the ocular surface: a review epithelial tumors of the ocular surface. Indian J Ophthalmol. 2015;63(2):187-203.

5 Rathi SG, Ganguly Kapoor A, Kaliki S. Ocular surface squamous neoplasia in HIV-infected patients: current perspectives. HIV AIDS. 2018 Mar 14;10:33-45.

6 Emmanuel B, Ruder E, Lin S-W, Abnet C, Hollenbeck A, Mbulaiteye S. Incidence of squamous-cell carcinoma of the conjunctiva and other eye cancers in the NIH-AARP Diet and Health Study. Ecancermedicalscience. 2012 May 21;6:254.

7 Onwubuya IM, Owoyele TM, Olaofe OO, Ezike KN. Morphological spectrum of orbitoocular diseases in a Tertiary Health Centre in Keffi, North Central Nigeria. Adv Med. 2015 Oct 20;2015:619414.
8 Alves LFDA, Fernandes BF, Burnier JV, Zoroquiain $\mathrm{P}$, Eskenazi DT, Burnier MN Jr. Incidence of epithelial lesions of the conjunctiva in a review of 12,102 specimens in Canada (Quebec). Arq Bras Oftalmol. 2011;74(1):213.

9 García Onrubia L, Pacheco-Callirgos GE, Portero-Benito A, García-Álvarez C, Carreño Salas E, Muñoz-Moreno MF, et al. Spectrum of conjunctival tumours in a Spanish series: a review of 462 cases. Eur J Ophthalmol. 2020; 30(6):1403-9.

10 Amoli FA, Heidari AB. Survey of 447 patients with conjunctival neoplastic lesions in Farabi Eye Hospital, Tehran, Iran. Ophthalmic Epidemiol. 2006 Jan 1;13(4):275-9.

11 Lee GA, Hirst LW. Ocular surface squamous neoplasia. Surv Ophthalmol. 1995 May 1; 39(6):429-50.

12 Basti S, Macsai MS. Ocular surface squamous neoplasia: a review. Cornea. 2003;22(7):687704.

13 Adesina A, Chumba D, Nelson AM, Orem J, Roberts DJ, Wabinga H, et al. Improvement of pathology in sub-Saharan Africa. Lancet Oncol. 2013;14(4):152-7.

14 Rambau PF. Pathology practice in a resourcepoor setting: Mwanza, Tanzania. Arch Pathol Lab Med. 2011 Feb 1;135(2):191-3.

15 Amin MB, Edge S, Greene F, Byrd DR, Brookland RK, Washington MK, et al. AJCC cancer staging manual. 8th ed. Berlin, Germany: Springer; 2018. p. 1032.
16 Josephat PK, Ame A. Effect of testing logistic regression assumptions on the improvement of the propensity scores. Int J Stat Appl. 2018; 8(1):9-17.

17 Shields CL, Alset AE, Boal NS, Casey MG Knapp AN, Sugarman JA, et al. Conjunctival tumors in 5,002 cases. Comparative analysis of benign versus malignant counterparts. The 2016 James D. Allen Lecture. Am J Ophthalmol. 2016;173:103-33.

18 Mahoney MC, Burnett WS, Majerovics A, Tanenbaum H. The epidemiology of ophthalmic malignancies in New York State. Ophthalmology. 1990;97(9):1143-7.

19 Yu GP, Hu DN, McCormick S, Finger PT. Conjunctival melanoma: is it increasing in the United States? Am J Ophthalmol. 2003; 135(6):800-6.

20 Cardenas-Cantu E, Zavala J, Valenzuela J, Valdez-García JE. Molecular basis of pterygium development. Semin Ophthalmol. 2016; 31(6):567-83.

21 Kheir WJ, Tetzlaff MT, Pfeiffer ML, Mulay K, Ozgur O, Morrell G, et al. Epithelial, non-melanocytic and melanocytic proliferations of the ocular surface. Semin Diagn Pathol. 2016; 33(3):122-32.

22 Mikropoulos D, Mavrikakis I, Ziakas NG, Konstas AG, Boboridis KG. Kaposi's sarcoma of the bulbar conjunctiva in an immunocompetent patient. Case Rep Ophthalmol. 2011; 2(2):193-7. 
23 Maia S, Gomes M, Oliveira L, Torres P. Isolated bulbar conjunctival Kaposi's sarcoma as a primary presentation of AIDS: a case report. Case Rep Ophthalmol Med. 2013;2013: 469195.

24 Murray N, McCluskey P, Wakefield D, Beaumont $\mathrm{P}$. Isolated bulbar conjunctival Kaposi's sarcoma. Aust N Z J Ophthalmol. 1994;22(1): 81-2.

25 Feng L, Cai D, Muhetaer A, Yang YL, Ren F, Yishake M, et al. Spindle cell carcinoma: the general demographics, basic clinico-pathologic characteristics, treatment, outcome and prognostic factors. Oncotarget. 2017;8(26): 43228-36.

26 Sun EC, Fears TR, Goedert JJ. Epidemiology of squamous cell conjunctival cancer. Cancer Epidemiol Biomarkers Prev. 1997;6(2):73-7.

27 Furahini G, Lewallen S. Epidemiology and management of ocular surface squamous neoplasia in Tanzania. Ophthalmic Epidemiol. 2010;17(3):171-6.

28 UNAIDS, Aidsinfo.unaids.org. UNAIDS Data 2019 [Internet]. Reference. 2019 [cited 2020 Jan 3].p. 1-476. Available from: https:// www.unaids.org/sites/default/files/media asset/2019-UNAIDS-data_en.pdf.

29 Yousef YA, Finger PT. Squamous carcinoma and dysplasia of the conjunctiva and cornea: an analysis of 101 cases. Ophthalmology. 2012;119(2):233-40.

30 Cervantes G, Rodríguez AA Jr, Leal AG. Squamous cell carcinoma of the conjunctiva: clinicopathological features in 287 cases. Can J Ophthalmol. 2002;37(1):14-20.

31 Pyne JH, Myint E, Barr EM, Clark SP, David $\mathrm{M}, \mathrm{Na}$ R. Acantholytic invasive squamous cell carcinoma: tumor diameter, invasion depth, grade of differentiation, surgical margins, perineural invasion, recurrence and death rate. J Cutan Pathol. 2017;44(4):320-7.
32 Yorita K, Tsuji K, Takano Y, Kuroda N, Sakamoto K, Arii K, et al. Acantholytic squamous cell carcinoma of the lung with marked lymphogenous metastases and high titers of myeloperoxidase-antineutrophil cytoplasmic antibodies: a case report. BMC Cancer. 2018; 18(1):300.

33 Ozer EE, Soydemir GP. Acantholytic squamous cell carcinoma of uncommon sites. Adv Cancer Res Clin Imaging. 2019;1(4):1-3.

34 Chernock RD, El-Mofty SK, Thorstad WL, Parvin CA, Lewis IS. HPV-related nonkeratinizing squamous cell carcinoma of the oropharynx: utility of microscopic features in predicting patient outcome. Head Neck Pathol. 2009;3(3):186-94.

35 El-Mofty SK, Zhang MQ, Davila RM. Histologic identification of human papillomavirus (HPV)-related squamous cell carcinoma in cervical lymph nodes: a reliable predictor of the site of an occult head and neck primary carcinoma. Head Neck Pathol. 2008;2(3): 163-8.

36 Fujimaki M, Fukumura Y, Mitani K, Kurisaki A, Yokoyama J, Ikeda K, et al. Histological subtypes and characteristic structures of HPV-associated oropharyngeal carcinoma; study with Japanese cases. Diagn Pathol. 2013;8(1):211.

37 Makupa II, Swai B, Makupa WU, White VA, Lewallen S. Clinical factors associated with malignancy and HIV status in patients with ocular surface squamous neoplasia at Kilimanjaro Christian Medical Centre, Tanzania. Br J Ophthalmol. 2012 Apr 1;96(4):482-4.

38 Kamal S, Kaliki S, Mishra DK, Batra J, Naik MN. Ocular surface squamous neoplasia in 200 patients: a case-control study of immunosuppression resulting from human immunodeficiency virus versus immunocompetency. Ophthalmology. 2015;122(2):1688-94.

39 Pola EC, Masanganise R, Rusakaniko S. The trend of ocular surface squamous neoplasia among ocular surface tumour biopsies submitted for histology from Sekuru Kaguvi Eye Unit, Harare between 1996 and 2000. Cent Afr J Med. 2003;49:1-2.
40 Bradley JC, Yang W, Bradley RH, Reid TW, Schwab IR. The science of pterygia. Br J Ophthalmol. 2010;94(7):815-20.

41 Oellers P, Karp CL, Sheth A, Kao AA, Abdelaziz A, Matthews JL, et al. Prevalence, treatment, and outcomes of coexistent ocular surface squamous neoplasia and pterygium. Ophthalmology. 2013;120(3):445-50.

42 Mendoza PR, Craven CM, Ip MH, Wilson MW, Coroneo MT, Grossniklaus HE. Conjunctival squamous cell carcinoma with corneal stromal invasion in presumed pterygia: a case series. Ocul Oncol Pathol. 2018;4(4): 240-9.

43 Carreira H, Coutinho F, Carrilho C, Lunet N. HIV and HPV infections and ocular surface squamous neoplasia: systematic review and meta-analysis. Br J Cancer. 2013 Oct 1;109(7): 1981-8.

44 Gichuhi S, Sagoo MS, Weiss HA, Burton MJ. Epidemiology of ocular surface squamous neoplasia in Africa. Trop Med Int Health. 2013 Dec;18(12):1424-43.

45 Newton R, Reeves G, Beral V, Ferlay J, Parkin D. Effect of ambient solar ultraviolet radiation on incidence of squamous-cell carcinoma of the eye. Lancet. 1996 May 25;347(9013): $1450-1$

46 Guech-Ongey M, Engels E, Goedert JJ, Biggar RJ, Mbulaiteye S. Elevated risk for squamous cell carcinoma of the conjunctiva among adults with AIDS in the United States. Int J Cancer. 2008;122(11):2590-3.

47 Masanganise R, Mukome A, DariJ, MakunikeMutasa R. Bilateral HIV related ocular surface squamous neoplasia: a paradigm shift. Cent Afr J Med. 2010;56(5-8):23-6.

48 Winward KE, Curtin VT. Conjunctival squamous cell carcinoma in a patient with human immunodeficiency virus infection. Am J Ophthalmol. 1989;107(5):554-5. 